\title{
PHOTOBIOMODULATION 810 NM DIODE LASER AND FORMOCRESOL FOR PRIMARY MOLAR PULPOTOMY: A RANDOMIZED CLINICAL TRIAL
}

\author{
Alaa Nadhreen *, Heba Sabbagh ${ }^{* *}$, Najlaa Al-amoudi ${ }^{* * *}$ and Heba Elkhodary ${ }^{* * * *}$
}

\begin{abstract}
Objectives: Low-level laser therapy (LLLT), commonly referred to as photobiomodulation therapy, has become a technique of interest due to its anti-inflammatory action. The aim of this study was to assess and compare the clinical and radiographic success rates of LLLT (PBM) 1 and formocresol (FC) for pulpotomy in human primary teeth.
\end{abstract}

Methods: This randomized split-mouth clinical trial included a sample of 106 primary molars from 36 children aged between 5-8 years. Selected teeth were equally assigned to two groups: LLLT (PBM) and FC groups. The treated teeth were evaluated clinically after 3 and 9 months and radiographically after 9 months. Mc-Nemar test was used to compare the outcomes between groups.

Results: After 3 and 9 months, the clinical success rates were $98 \%$ for both groups. The radiographic success rates were 100\% in the LLLT (PBM) group and 98\% for the FC group at the 9-month follow-up interval.

Conclusions: Our results suggest that LLLT (PBM) yielded favorable initial outcomes when compared to FC in human primary molar teeth over a 9-month follow-up period. Further studies with a longer follow-up period is still required in addition to histological studies for the potential healing effects of LLLT (PBM) to support the initial clinical and radiographic outcomes.

Trial Registration: This trial was retrospectively registered at ClinicalTrials.gov under the ID, NCT03782714, on 22 ${ }^{\text {nd }}$ of December 2018.

KEYWORDS: Low-level laser therapy, photobiomodulation, pulpotomy, primary molars, formocresol, $810 \mathrm{~nm}$ diode laser.

\footnotetext{
* Teacher Assistant, Pediatric Dentistry Department, Faculty of Dentistry, King Abdulaziz University, Jeddah, Saudi Arabia. ** Assistant Professor, Pediatric Dentistry Department, Faculty of Dentistry, King Abdulaziz University, Jeddah, Saudi Arabia *** Professor, Pediatric Department, Faculty of Dentistry, King Abdulaziz University, Jeddah, Saudi Arabia.

**** Associate Professor, Pediatric Dentistry Department, Faculty of Dentistry, King Abdulaziz University, Jeddah, Saudi Arabia; (Corresponding author) Assistant Professor, Pediatric Dentistry Department, Faculty of Dental Medicine for Girls, Al-Azhar University, Cairo, Egypt.
} 


\section{INTRODUCTION}

Dental caries is an infectious, multifactorial disease that remains an important public health concern worldwide. ${ }^{1}$ When the disease is left untreated, it progresses through the enamel and dentine and reaches the pulp of the tooth. If the inflammation caused by the progression of dental caries is within the confines of the coronal portion of the pulp, the treatment of choice in children would be pulpotomy that involves the removal of the inflamed coronal pulp while maintaining the radicular pulp intact using different pulp dressing materials. $^{2}$

Formocresol (FC) was introduced by Buckly in 1904, and since then the pulpotomy protocol for 1:5 dilution FC has become the standard practice for pulpotomy. ${ }^{3}$ Several other products have been proposed for primary teeth pulpotomy, such as glutaraldehy, electrosurgery, laser, bioactive glass, taking into account the local effect and systemic absorption of FC. All of them have shown a varied amount of success. ${ }^{4,5}$ Though a lot of debate exists regarding FC adverse effects being carcinogenic, cytotoxic, and mutagenic, UK National Clinical Guidelines in Pediatric Dentistry have proposed FC in 1:5 dilution as one of the choices of materials recommended for primary tooth pulpotomy. ${ }^{6}$

Nowadays, the use of laser not only invaded the medical field but also it has many applications in the dental practice. Different types of laser had been investigated in many animal and human studies and reported promising results regarding hemostasis, absence of mechanical contact and stimulation of regeneration. ${ }^{7}$ In the pediatric dental sector, laser use continues to produce promising effects. ${ }^{8}$ Children are still waiting for a comfortable, pleasing dental experience as they seek dental treatment. They usually dislike heat, vibration, noise, bleeding and unpleasant smells and tastes.. Laser is a safe alternative for decreasing the likelihood of what could be a psychologically harmful memory in a child mind. Basically speaking, children are more tolerant of treatment and more cooperative during laser-aided dental procedures. ${ }^{10}$ Researchers are more encouraged to use various types of laser in a pulpotomy procedure owing to the property of decreasing bacterial count in human tissues. Back in 1985, Shoji was the first to apply laser in pulpotomy procedure wher they examined the effects of $\mathrm{CO}_{2}$ laser application on dental pulp tissues of $\operatorname{dogs}{ }^{11}$ Ever Since, many studies investigated several other types of lasers on human primary teeth pulps. ${ }^{12-14}$ Beside the reported advantages being minimally invasive, less painful and a more comfortable technique, its use also eliminates the noise and vibrations and sometimes the use of injections. ${ }^{15}$ In 2018, a recent systematic review and meta-analysis was conducted to compare clinical and radiographic success rates of different types of laser pulpotomy with those of other pulpotomy techniques in primary teeth. It concluded that clinical and radiographic success rates of laser pulpotomy up to 18 months follow-up were comparable to other pulpotomy techniques, including MTA and FC. ${ }^{16}$

Low-level laser therapy (LLLT), commonly referred to as photobiomodulation (PBM) therapy, has become a technique of interest to many researchers due to its anti-inflammatory action, the ability to increase collagen production, reduce the inflammatory exudation and enhance revascularization and epithelization..$^{7,17-20}$ In addition, this technique is minimally invasive and consumes less time. It showed promising results when used in primary molar pulpotomy owing to its ability to enhance pulpal healing, reduce inflammatory process and maintain pulp vitality. ${ }^{7,21}$

Few studies examined LLLT's (PBM's) clinical and radiographic impact on primary teeth pulps using different types of laser, with varying wavelengths and energy dosages, resulting in conflicting evidence of LLLT's influence on the mechanism of pulp tissue repair. In 2010, Golapayegani et al., compared LLLT and FC and found that LLLT can be used successfully as a substitute to FC but longer 
follow up periods were recommended..$^{21}$ Durmus and Tanboga in 2014, conducted an in vivo study to evaluate the effects of Diode Laser on primary molars pulpotomy and compared its success to FC and FS. The differences though not significant, Diode Laser was not suggested as to replace FC based on the relatively low radiographic success rate..$^{22}$ In 2015, Fernandes et al., evaluated the effectiveness of LLLT on human primary teeth with vital pulps against $\mathrm{FC}$ and $\mathrm{CaOH}$. The study concluded LLLT as an adjunct alternative for pulpotomy. ${ }^{23}$ Only one study in 2015 evaluated the histological effects of LLLT, Buckley's FC (diluted at 1:5) and calcium hydroxide on the pulps of primary molars. The results concluded that LLLT exhibited satisfactory performance in the pulp healing process and recommended further clinical studies on human teeth with longer follow-up periods. ${ }^{24}$ Uloopi et al. in 2016 compared LLLT and MTA in primary molar pulpotomy. Following twelve months evaluation, MTA demonstrated a success rate of $94.7 \%$ at all intervals, while LLLT showed $95 \%$ success rate at 3 months, which declined gradually to $85 \%$ at 6 months, reaching $80 \%$ at 12 months. However, the intergroup comparison was insignificant and LLLT success was comparable to MTA. ${ }^{25}$

Based on the literature above, the information on the success rate of LLLT (PBM) in pulpotomy is limited, and conflicting results have been reported. There is a lack of knowledge on the best dosages to be used for biological pulp healing. Therefore, further research should be conducted in order to standardize this technique as an adjunct alternative for vital pulp therapy on human primary teeth and to develop a clearer idea regarding its mechanism of action. Thus, in this part of the study, the aim was to assess and compare the clinical and radiographic success rates of LLLT (PBM) and FC for pulpotomy in human primary teeth. The study's hypothesis was that there would be no difference in the pulpotomy of primary teeth between the two groups.

\section{MATERIALS AND METHODS}

This randomized controlled clinical split-mouth blinded study was conducted at the Faculty of Dentistry of King Abdulaziz University in Jeddah (from August 1, 2016 to August 31, 2017). It was approved by the Research Ethics Committee of the Faculty of Dentistry in the year 2016 under the approval number (099-11-17) and registered on ClinicalTrials.gov Identifier number (NCT03782714). The sample size for this randomized control trial was measured using Open Source Epidemiologic Statistics for Public Health online software (www.openepi.com). It was calculated using estimates provided in previous reports and indicating about $20 \%$ difference in success rate between LLLT and FC groups $(78.8 \%$ vs $57.8 \%$; and $71.42 \%$ vs $90.47 \%$ ). ${ }^{26}$ Sample size calculated based on averages of these estimates indicated that a sample size of 51 teeth in the test group and 51 teeth in the control group was necessary for $80 \%$ power, which is considered an adequate power level.

After obtaining written informed consents from the patients' parents, one experienced pediatric dentist recruited children aged between 5-8 years. Each child had at least 2 contralateral first or second primary molars that were indicated for pulpotomy. The included children were physically and mentally healthy without any known medical history of systemic conditions contraindicating pulp therapy. The selected children had "positive" or "definitely positive" behavioral ratings according to the Frankl behavior classification scale. ${ }^{27}$ The inclusion criteria for the teeth were as follows: restorable crowns with vital carious pulp exposure; no clinical signs and symptoms of pulp degeneration, such as swelling, fistula, abnormal mobility, spontaneous pain, or sensitivity to percussion; no radiographic signs such as those indicating internal or external resorption and periapical or interradicular radiolucency; and less than $1 / 3$ of the root resorbed normally. 
Since a split-mouth study design was utilized, it was specified to start with the right side despite the technique chosen by randomization. Therefore, randomization was carried out for the technique only. Before recruitment started, 53 sealed envelopes containing the randomization results were prepared, sealed and blindly mixed in a box. Each envelope represented a pair of matched contralateral teeth. Then these envelopes were numbered from 1 to 53 . The number of each envelope also determined the pair order in the sequence of treatment (envelope number 1 indicated the first treated pair and so on). Each envelope was un-sealed after the parent signed the informed consent and immediately, by the operator, before pulpotomy procedure was performed.

The same pediatric dentist who recruited the children performed all pulpotomies. All the teeth were anesthetized using 27 -gauge short needles and syringes loaded with carpules containing $1.8 \mathrm{ml}$ of lidocaine $\mathrm{HCl} 2 \%$ and an epinephrine concentration of 1:100000. Isolation was carried out using a rubber dam and an appropriate clamp. Subsequently, the pulpotomy procedure was performed, which included caries excavation using a no. 330 highspeed bur with a water spray and coronal pulp amputation using a sharp spoon excavator or a slow-speed round carbide bur (no. 6 or no. 8). Following this, the pulp chamber was irrigated with distilled water, and the hemorrhage was controlled by placing a cotton swab that was moistened with normal saline in the pulp chamber for 5 minutes.

In LLLT (PBM) group, safety goggles were worn by the operator, patient, and parent. Subsequently, laser radiation with a wavelength of $810 \mathrm{~nm}$ (Photon Dental Diode Laser®, Zolar Technology \& Mfg. Co. Inc., Mississauga, Ontario, Canada) was delivered through a $200-\mu \mathrm{m}$-diameter optical fiber. The fiber tip was kept $2 \mathrm{~mm}$ away from touching the pulp tissues. The laser parameters were set at the following low-level settings on the biosimulation program: $3 \mathrm{~W}$ power output, $5 \mathrm{~W} / \mathrm{cm}^{2}$ power density,
4 Joules energy, $6.7 \mathrm{~J} / \mathrm{cm}^{2}$ energy density, 1-50 $\mathrm{KHz}$ frequency, $105-\mu \mathrm{m}$ focus beam diameter, and an irradiation time of $40 \mathrm{~s}$ (per tooth) continuously with air-cooling operation mode without water. The tip of the laser device was rotated over all the pulp stumps during application. Table 1 outlines the device information, the irradiation and treatment parameters. In the FC group, a cotton pellet moistened with 1:5 dilution of FC (Formocresol ${ }^{\circledR}$, Viarden Dental, Marcelino Dávalo, Algarín, Mexico) was placed on the amputated pulp for 5 minutes. Subsequently, the pulp chambers of all the teeth were filled with reinforced zinc oxide-eugenol (IRM®), Dentsply, Gilby Rd, Mount Waverley, Australia) to ensure proper sealing. ${ }^{23,24}$

In both groups, each tooth was finally restored with a stainless steel crown (SSC) (3M/ESPE, St. Paul, Minn., USA). Following the pulpotomy procedure, during the same visit, each molar was prepared, and the crown was fitted and cemented onto the tooth using glass ionomer cement (Rely-X 3M/ESPE, St. Paul, Minn., USA). Finally, a postoperative periapical radiograph was captured for each tooth in order to confirm that the remaining pulp tissues were properly dressed. The parents and children were provided with the required general oral hygiene instructions in relation to the treated teeth. Moreover, all the possible outcomes were explained to them in detail, and they were asked to immediately report any pain, discomfort, swelling, or pus discharge.

For ethical issues, the radiographic examination before 9 months was performed only if patients complained. If postoperative pain was reported, and the examination revealed improperly seated crown or long margins, the decision would be crown replacement and the case would be successful. However, if the pain was related to the crown with normal radiographic findings, the oral hygiene instruction would be reinforced and the patient would be followed-up and that would also be considered a successful case. 
TABLE (1) Laser device* irradiation and treatment parameters according to the manufacturer's instructions

\begin{tabular}{|c|c|}
\hline \multicolumn{2}{|l|}{ Device information } \\
\hline Manufacturer & Zolar Technology \& Mfg. Co. Inc. \\
\hline Model Identifier & Ph031501081 \\
\hline Year Produced & 2015 \\
\hline Beam Delivery System & Fiberoptic \\
\hline Irradiation parameter [unit] & Value, Measurement method, or Information source \\
\hline Center wavelength $[\mathrm{nm}]$ & 810 \\
\hline Operating mode & Continuous or pulsed wave \\
\hline Frequency $[\mathrm{Hz}]$ & $0.1-50 \mathrm{KHz}$ \\
\hline Pulse on duration $[\mathrm{sec}]$ & $0.01 \mathrm{~ms}$ to $9.9 \mathrm{~s}$ \\
\hline Pulse off duration [sec] or duty cycle [\%] & Variable \\
\hline Energy per pulse $[\mathrm{J}]$ & 4 \\
\hline Average radiant power $[\mathrm{mW}]$ & 2 \\
\hline Beam profile & Gaussian \\
\hline Treatment parameter [unit] & Value \\
\hline Beam spot size at target $\left[\mathrm{cm}^{2}\right]$ & $105 \mu \mathrm{m}$ \\
\hline Exposure duration [sec] & 40 \\
\hline Radiant exposure $\left[\mathrm{J} / \mathrm{cm}^{2}\right]$ & 6.7 \\
\hline Radiant energy [J] & 4 \\
\hline Number of points irradiated & 1 \\
\hline Application technique & Non-contact mode \\
\hline Number and frequency of treatment sessions & 1 session \\
\hline Total radiant energy $[\mathrm{J}]$ & 4 \\
\hline
\end{tabular}

*Photon Dental Diode Laser ${ }^{\circledR}$, Zolar Technology \& Mfg Co. Inc., Mississauga, Ontario, Canada.

nm: nanometer, Hz: hertz, KHz: kilohertz, s: second, ms: millisecond, \%: percentage, J: joule, mW: Milliwatt, cm2: square, centimeter, $\mu m$ : micrometer, J/cm2: joules per square centimetre.

\section{Follow up:}

All the included patients were recalled after 3 months for a clinical assessment and after 9 months for both clinical and radiographic evaluations. The same operator who performed all the pulpotomy procedures clinically and radiographically evaluated the treated teeth. To confirm intra-examiner reliability, 1 week following the initial evaluation, the examiner re-evaluated the radiographs of 10 cases, and there were no differences in results between both the evaluations. During the clinical and radiographic evaluations, the examiner was blinded to the group allocation. 


\section{Outcome assessment criteria:}

The outcome of success or failure was determined by the following clinical and radiographic criteria. The treatment was considered a clinical failure if the tooth presented with any of the following clinical signs or symptoms: pain; swelling; sinus tract; mobility; or pain on percussion. The treatment was considered a radiographic failure if the periapical radiograph showed: internal root resorption; furcation radiolucency; periapical radiolucency; or widening of the periodontal ligament space. Any tooth that had a treatment failure received proper treatment tailored to that particular tooth. ${ }^{28-31}$ The intra-examiner reliability for clinical examination and radiographic evaluation were $100 \%$ with Cohen kappa coefficient of 1 ( $\mathrm{p}<0.001)$.

\section{Statistical analysis:}

Data were analyzed using the Statistical Package for Social Sciences v 16.0 (SPSS Inc., Chicago, IL, USA). Descriptive statistics were presented as frequency and percentages. McNemar test was utilized to test significant differences between the study and control group for clinical and radiographic outcomes. The level of significance was set at $P<$ 0.05 . For significant $P$ values, Relative Risk (RR) and $95 \%$ confidence intervals (CI) were calculated.

\section{RESULTS}

The sample consisted of 106 molars in 36 patients ( $52.8 \%$ boys and $45.3 \%$ girls; mean age: $6.18 \pm 0.99$ years old). They were equally and randomly assigned to either the LLLT or the FC groups, using a split-mouth approach. Two patients (with four molars) failed to attend the 3-month follow-up after moving to distant cities. These teeth were dropped from the data for the 3- and 9-month examinations and analysis. Therefore, a total of 102 molars were available for analysis. A CONSORT diagram showing the flow of patients and pulpotomized teeth up to the 9-month follow-up was presented in Figure 1. Table 2 presents the distribution of children as

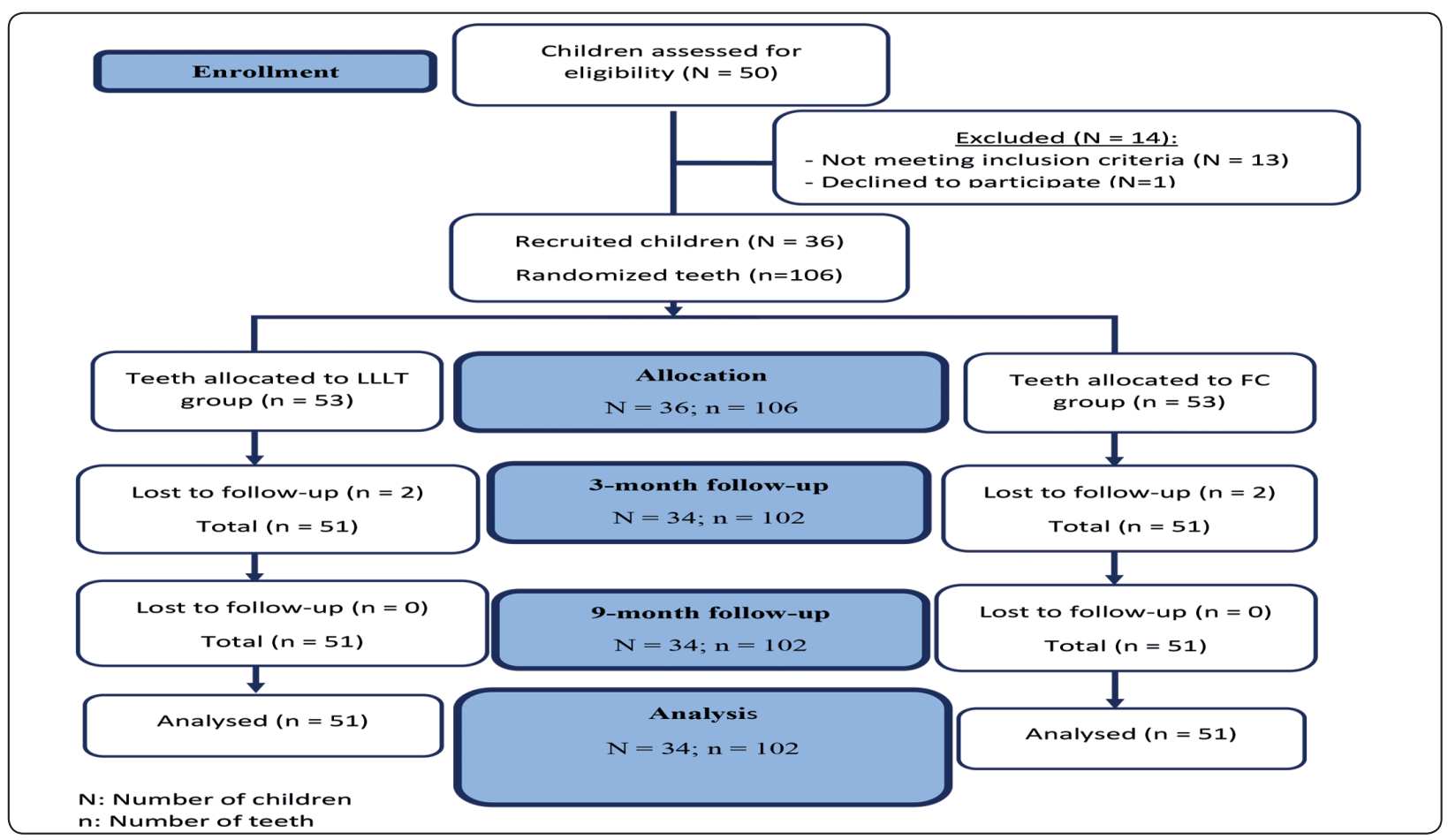

Fig. (1): Flow chart diagram for the study participants according to Consolidated Standards of Reporting Trials (CONSORT) guidelines 
follows: 23 children $(63.9 \%)$ had a single pair of teeth, 10 children $(27.8 \%)$ had two pairs of teeth, two children (5.6\%) had three pairs of teeth and one child $(2.7 \%)$ had four pairs of teeth.

TABLE (2) Characteristics of the included children

\begin{tabular}{|c|c|c|c|}
\hline \multirow{2}{*}{ Item } & \multirow{2}{*}{ Category } & \multicolumn{2}{|c|}{$\begin{array}{c}\text { Total number of } \\
\text { children }\end{array}$} \\
\hline & & $\mathbf{N}^{*}$ & $\%$ \\
\hline \multirow{4}{*}{$\begin{array}{c}\text { Number } \\
\text { of pairs of } \\
\text { teeth* }\end{array}$} & Single pair of teeth & 23 & 63.9 \\
\hline & Two pairs of teeth & 10 & 27.8 \\
\hline & Three pairs of teeth & 2 & 5.6 \\
\hline & Four pairs of teeth & 1 & 2.7 \\
\hline \multirow{2}{*}{ Sex } & Male & 19 & 52.8 \\
\hline & Female & 17 & 47.2 \\
\hline \multirow{4}{*}{ Age (years) } & 5 & 11 & 30.6 \\
\hline & 6 & 12 & 33.3 \\
\hline & 7 & 8 & 22.2 \\
\hline & 8 & 5 & 13.9 \\
\hline
\end{tabular}

*N: Number of children (23 children had a single pair of teeth $=23 \times 2=46$ teeth,

10 children had two pairs of teeth $=10 x(2 \times 2)=40$ teeth,

2 children had 3 pairs of teeth $=2 x(3 \times 2)=12$ teeth,

1 child had four pairs of teeth $=1 \times(4 \times 2)=8$ teeth $)$

Table 3 shows the clinical outcomes of LLLT and FC groups during study follow up periods. At the 3-month follow-up, LLLT (PBM) and FC resulted in an overall clinical success rate of $98 \%$. Postoperative pain was reported in two molars: one from each group. No statistically significant differences were noted between the groups at the 3-months follow-up. For the molar in the LLLT group, radiographic evaluation was performed showing normal findings. The molar was diagnosed to have referred pain from the adjacent molar. Once the molar was restored, the pain had gone, and the child no longer complained. In the FC group, a periapical radiograph was taken revealing normal findings, while clinical examination showed that the crown margins were long. The crown was replaced, and the pain subsided. At the 9-month follow-up, 51 teeth in each of the LLLT and FC groups were available for clinical evaluation. Both groups showed a $98 \%$ clinical success rate. One primary molar from the LLLT group had non-spontaneous pain as well as grade one mobility, while one in the FC group experienced non-spontaneous pain only.

The radiographic outcomes of LLLT and FC groups at the 9-month follow-up were represented in Table 4. Fifty-one teeth were available for radiographic examination in each of the LLLT and FC groups. For the LLLT and FC groups, the radiographic success rates were 100 percent and 98 percent respectively. Furcation involvement was identified in one primary molar from the FC group. At the 9-month follow-up there were no statistically significant discrepancies between the two groups. The tooth with grade one mobility in the LLLT group was closely monitored, as it was asymptomatic. The tooth in the FC group showed signs of furcation involvement. Later, the parent reported the presence of an abscess related to that tooth and it was extracted. Figures 2 shows a periapical radiograph after a successful LLLT (PBM) pulpotomy while figure 3 presents a radiograph of a successful FC pulpotomy. 
TABLE (3) Clinical and radiographic outcomes of treatment groups during study follow-up periods

\begin{tabular}{|c|c|c|c|c|c|c|c|c|c|c|c|}
\hline \multirow{3}{*}{ Follow up } & \multicolumn{5}{|c|}{ LLLT } & \multicolumn{5}{|c|}{ FC } & \multirow{3}{*}{$\begin{array}{c}P \text { value } \\
\text { OR } \\
(95 \% \mathrm{CI})\end{array}$} \\
\hline & \multirow{2}{*}{$\mathbf{N}$} & \multicolumn{2}{|c|}{ Success } & \multicolumn{2}{|c|}{ Failure } & \multirow{2}{*}{$\mathbf{N}$} & \multicolumn{2}{|c|}{ Success } & \multicolumn{2}{|c|}{ Failure } & \\
\hline & & n & $\%$ & $\mathbf{n}$ & $\%$ & & $\mathbf{n}$ & $\%$ & $\mathbf{n}$ & $\%$ & \\
\hline \multicolumn{12}{|c|}{ Clinical outcomes } \\
\hline 3 mos. & 51 & 50 & 98 & 1 & 2 & 51 & 50 & 98 & 1 & 2 & $\begin{array}{c}1.0 \\
1.0 \\
(0.064-15.56)\end{array}$ \\
\hline 9 mos. & 51 & 50 & 98 & 1 & 2 & 51 & 50 & 98 & 1 & 2 & $\begin{array}{c}1.0 \\
1.0 \\
(0.064-15.56)\end{array}$ \\
\hline \multicolumn{12}{|c|}{ Radiographic outcomes } \\
\hline 9 mos. & 51 & 51 & 100 & 0 & 0 & 51 & 50 & 98 & 1 & 2 & $\begin{array}{c}0.317 \\
1.02 \\
(0.98-1.06)\end{array}$ \\
\hline
\end{tabular}

Mos.: Months

n: Number of successful and failed teeth in each group

FC: Formocresol

CI: Confidence Interval
$N$ : Total number of treated teeth in each group LLLT: Low-Level Laser Therapy RR: Relative Risk

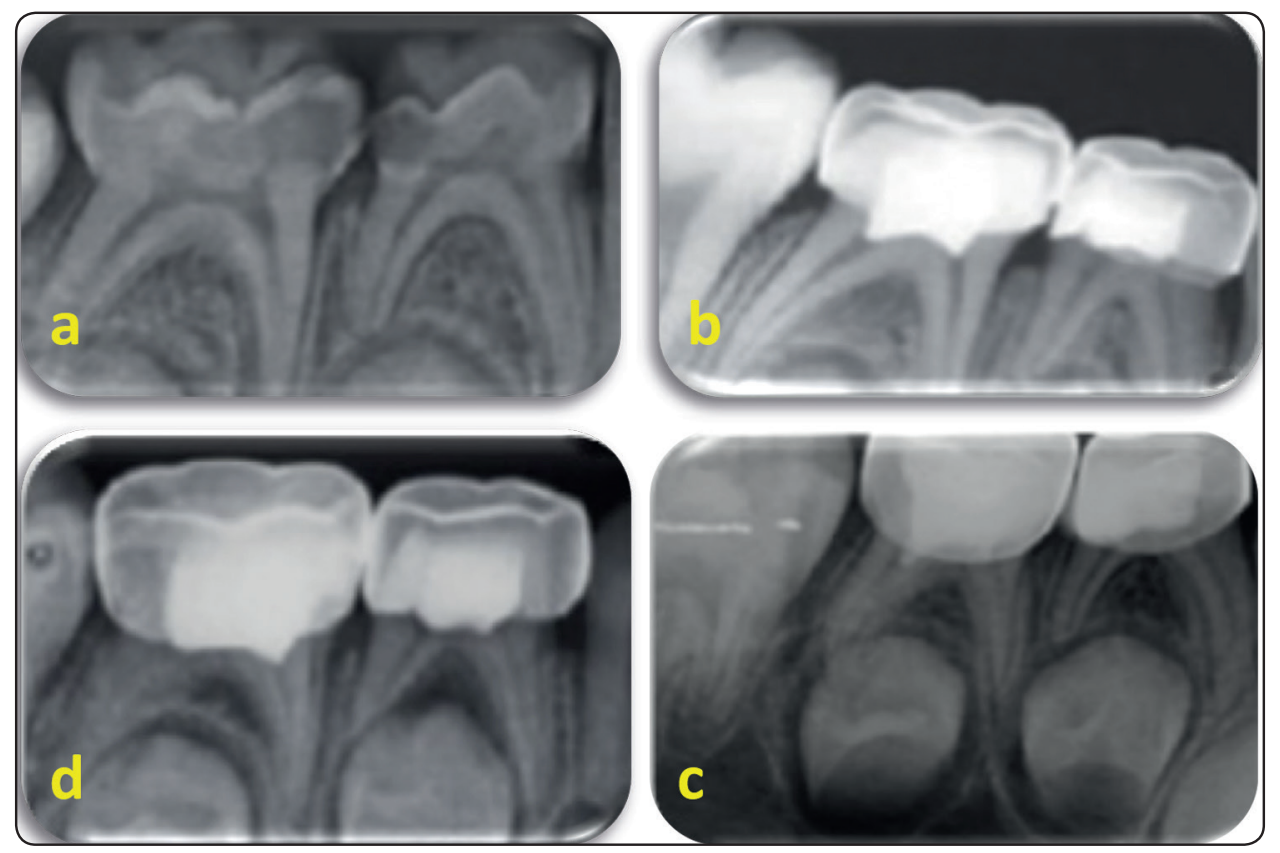

Fig. (2) Periapical radiographs showing a successful low-level laser therapy pulpotomy in teeth \#84 and \#85. a) Preoperative radiograph. b) Postoperative radiograph. c) 3-month follow-up radiograph. d) 9-month follow-up radiograph. 


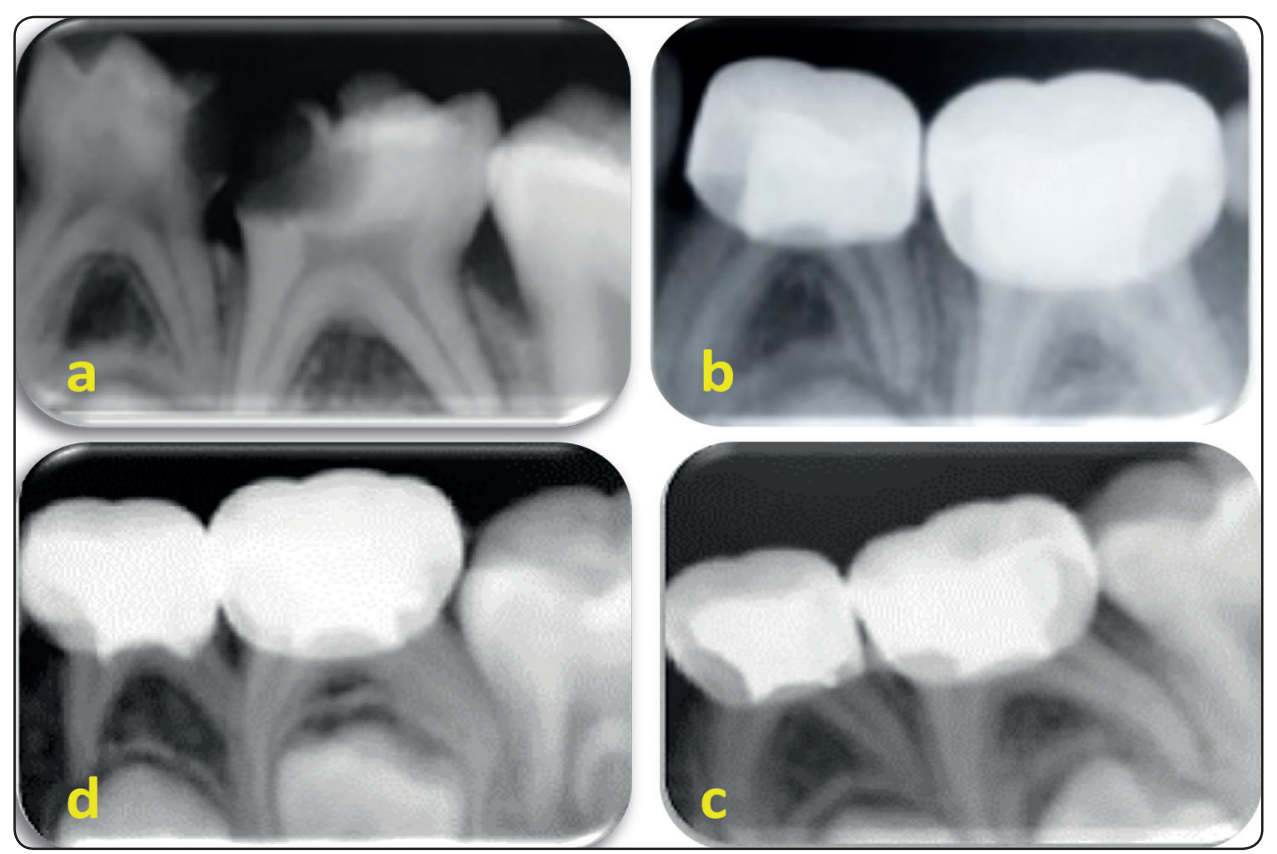

Fig. (3) Periapical radiographs showing a successful formocresol pulpotomy in teeth \#74 and \#75. a) Preoperative radiograph. b) Postoperative radiograph. c) 3-month follow-up radiograph. d) 9-month follow-up radiograph.

\section{DISCUSSION}

Pulpotomy is a pulp therapy modality that has the advantage of preserving the vitality of the primary pulp until the time of normal exfoliation. Many agents and techniques have been implicated for the pulpotomy procedure, with formocresol being the standard. ${ }^{32,33}$ In this study, LLLT (PBM) was assessed as a new trend for the pulpotomy procedure showing a clinical and radiographic success rate similar to formocresol. However, the actual healing effect of LLLT (PBM) on the pulpal tissues would be tested histologically in the second part of our project.

The methodology used in this study along with the follow-up intervals were similar to other clinical trials that investigated the clinical and radiographic success rates of LLLT (PBM) using different laser device parameters. ${ }^{21-25}$ However, the number of subjects included in the current study was larger and based on statistical calculation. The laser energy (4 J) used in our study was according to Sun and Tuner (2004) who set a general rule for the use of
LLLT (PBM) intra-orally; indicating that the laser energy should range between 2-4 $\mathrm{J}$ to be used inside the oral cavity. ${ }^{34}$ The wavelength used in this study $(810 \mathrm{~nm})$ was similar to that used in the studies by and Durmus and Tamboga and Uloopi et al..$^{22,25}$ Golpayegani et al. used a wavelength of $632 \mathrm{~nm},{ }^{21}$ whereas Fernandes et al. and Marques et al. used a wavelength of $660 \mathrm{~nm}^{23,24}$ It was demonstrated that when the lasers are within the visible spectrum (600-700 nm wavelengths), and their energy den sity ranges between $0.5-4 \mathrm{~J} / \mathrm{cm}^{2}$, they would enhance cell proliferation and other cellular functions. On the other hand, wavelengths greater than $810 \mathrm{~nm}$ with energy densities higher than $10 \mathrm{~J} / \mathrm{cm}^{2}$ could have inhibitory effects. ${ }^{35,36}$ The presence of such variations in the parameters among studies and the lack of consensus on these parameters could still be a hindrance in approving the use of this technique as an alternative to FC despite its high success rates.

The success rate of pulpotomies has been measured traditionally as the percentage of teeth reaching an arbitrary point in time in the absence 
of clinical or radiographic evidence of disease..$^{25}$ The results of this study demonstrated high clinical success rates (98\%) of both LLLT (PBM) and FC groups during the 3 and 9-month follow-ups. The results are consistent with previous findings where Durmus and Tanboga and Fernandes et al. demonstrated a $100 \%$ clinical success rates for both LLLT (PBM) and FC. ${ }^{22,23}$ These findings also agree with Golpayegani et al. who reported a success rate of $100 \%$ for LLLT (PBM) and FC at six months follow-up. ${ }^{21}$ Only one study conducted by Uloopi et al. showed a less favorable success rate of $85 \%$ for LLLT (PBM) after 6 months follow-up. ${ }^{25}$ This might be due to the differences in LLLT (PBM) parameters used between the studies. Although a similar wavelength $(810 \mathrm{~nm})$ was used, Uloopi et al. applied LLLT (PBM) over radicular pulp stumps for only 10 seconds while in our study the time of application was 40 seconds according to manufacturer instructions. ${ }^{25}$ This is supported by Durmus and Tanboga who found out that any variability in the laser application parameters including exposure time might affect pulpotomy outcomes differently. ${ }^{22}$

Regarding the radiographic success rates, the success rates were $100 \%$ and $98 \%$ for LLLT (PBM) group and FC group, respectively. No statistically significant difference was found. Our findings coincide with Saltzman et al., who found that there was no statistically significant differences between both groups regarding the radiographic success rates. ${ }^{37}$ The results were different from those of Golapayegani et al. ${ }^{21}$ The differences of the findings between the two studies could be again related to variations in laser parameters used such as wavelength, power output, dose and pulse frequency. ${ }^{38}$ In the current study, LLLT (PBM) was used at $810 \mathrm{~nm}$ wavelength, 4 Joules energy and 40s application time under continuous mode. However, Golpayegani et al., used $632 \mathrm{~nm}$ wavelength under a continuous mode, with an energy of 4.0 $\mathrm{J} / \mathrm{cm}^{2}$ for about 30 seconds and his study reported a radiographical success rate of $67 \% .^{21}$ Also, our results do not agree with Fernandes et al. who reported $80 \%$ radiographic success rates of LLLT group. This could be due to the use of different sealing materials following pulpotomy. Fernandes et al. restored all teeth with resin-modified glass ionomer sealant after pulpotomy, ${ }^{23}$ while in our study, all teeth were covered with SSC to insure proper sealing and isolation.

Post-operative pain, which was seen in four teeth was the mostly reported clinical sign of failure in our study. This finding coincides with both Gupta et al. and Niranjani et al. who reported some cases demonstrating pain during the study recall visits..$^{39,40}$ However, Kuo et al. found that none of the teeth in the laser group had any clinical sign or symptom of failure, but their study was a retrospective one. ${ }^{14} \mathrm{In}$ addition to the fact that the laser pulpotomy is more operator-sensitive technique according to Niranjani et $a l,{ }^{40}$ the failure in the pulpotomy could be due to a variety of causes such as inappropriate crown adaptation, the presence of voids in the cement used, and areas of remaining caries or residual coronal pulp tissue. Furcation involvement was reported in only one case in the FC group at the 9 months follow up and the tooth was extracted, dropping the FC clinical success rate to $98 \%$. Also, in the FC group, abnormal mobility was also reported in one molar and it was planned for further followup. These findings agree with Ansari et al., who reported two cases with abnormal mobility one at 6 months and another at 12 months follow--ups and both cases were judged as failure. ${ }^{41}$ Almost all of the patients attended all follow-up visits resulting in low drop-out rate. However, a major limitation of this study is that one operator performed all pulpotomies and evaluated the teeth clinically and radiographically. A second limitation is that the type of post-operative pain should have been specified and described in more detail to clearly differentiate between pain that results from a tooth with a failed pulpotomy and that arises due to food impaction and bad oral hygiene. Another limitation is that the 
estimates that were based on sample size differed from the success rates that we eventually obtained after analysis. Owing to more moderate output of FC than that observed in the present analysis, the calculations we used for sample size measurement presumed a bigger difference between groups. Our results favour the null hypothesis but there is a possibility of under powering the research. We performed a post-hoc power review to determine if there was adequate power to sustain an argument that LLLT was not inferior to FC (using non-inferiority margin $=10 \%)$ and the findings showed power $>$ 98\% (http://powerandsamplesize.com/Calculators/ Compare-2-Proportions/2-Sample-Non-Inferiorityor-Superiority) and (https://www2.ccrb.cuhk.edu. hk/stat/proportion/tspp_sup.htm\#2). Our findings, thus, do not support that LLLT is better than FC although the claim that its success rate is not inferior to FC within $10 \%$ can be supported.

\section{CONCLUSIONS}

\section{Based on this study's results, the following conclusion can be made:}

1- LLLT (PBM) yielded favorable initial outcomes when compared to FC in human primary molar teeth over a 9-month follow-up period.

2- Further studies with a longer follow-up period is still required to aid in reaching a definitive conclusion regarding the success rates of LLLT (PBM).

3- Histological studies are also required to prove the potential healing effects of LLLT (PBM) to support the clinical and radiographic outcomes.

\section{ACKNOWLEDGEMENT}

This project was funded by the Deanship of Scientific Research (DSR) at King Abdulaziz University, Jeddah, under grant no. (G: 1408-1651440). The authors, therefore, acknowledge with thanks DSR for technical and financial support.

\section{REFERENCES}

1. Cummins D. (2013): Dental caries: a disease which remains a public health concern in the 21 st century--the exploration of a breakthrough technology for caries prevention. J Clin Dent., 24 Spec no A: A1-14.

2. Dhar V, Marghalani AA, Crystal YO, Kumar A, Ritwik P, Tulunoglu O, Graham L. (2017 ): Use of Vital Pulp Therapies in Primary Teeth with Deep Caries Lesions. Pediatr Dent., 15;39(5):146-159.

3. Chakraborty A, Dey B and Jana S. (2018): A Nonconventional Approach to Formocresol Pulpotomy. Int J Clin Pediatr. Dent., 11: 490.

4. Sant'anna AT, Spolidório LC and Ramalho LTO. (2008): Histological analysis of the association between formocresol and endotoxin in the subcutaneous tissue of mice. Braz Dent J., 19: 40-45.

5. Milnes AR. (2008): Is formocresol obsolete? A fresh look at the evidence concerning safety issues. Pediatr Dent., 30: 237-246.

6. Rodd H, Waterhouse P, Fuks A, Fayle S and Moffat M. (2006): Pulp therapy for primary molars. Int J Paediatr Dent., 16: 15-23.

7. De Coster P, Rajasekharan S and Martens L. (2013): Laser-assisted pulpotomy in primary teeth: a systematic review. Int J Paediatr Dent., 23: 389-399.

8. Prathima GS, Bhadrashetty D, Babu SB, Disha P. (2015): Microdentistry with lasers. J Int Oral Health.,7:134-7.

9. Kotlow L (2008): Lasers and pediatric dental care. Gen. Dent., 56: 618-627.

10. Nazemisalman B, Farsadeghi M, Sokhansanj M. (2015): Types of lasers and their applications in pediatric dentistry. J Lasers Med Sci., 6:96-101.

11. Shoji S, Nakamura M, Horiuchi H. (1985): Histopathological changes in dental pulps irradiated by $\mathrm{CO} 2$ laser: a preliminary report on laser pulpotomy. J Endod., 11:379-84.

12. Liu JF. (2006): Effects of Nd:YAG laser pulpotomy on human primary molars. J Endod., 32:404-7.

13. Huth KC, Hajek-Al-Khatar N, Wolf P, Ilie N, Hickel R, Paschos E. (2012): Long-term effectiveness of four pulpotomy techniques: 3-year randomised controlled trial. Clin Oral Investig., 16:1243-50.

14. Kuo H-Y, Lin J-R, Huang W-H and Chiang M-L. (2018): Clinical outcomes for primary molars treated by different types of pulpotomy: a retrospective cohort study. J Formos Med Assoc., 117: 24-33. 
15. Shanthi M. (2015): Laser Prescience in Pediatric Dentistry. Int. J. Sci. Study, 3: 197-203.

16. Nematollahi H., Sarraf Shirazi A., Mehrabkhani M., Sabbagh S. (2018): Clinical and radiographic outcomes of laser pulpotomy in vital primary teeth: a systematic review and meta-analysis. Eur Arch Paediatr Dent, 19: 205-220.

17. Olivi G, Genovese M and Caprioglio C. (2009): Evidencebased dentistry on laser paediatric dentistry: review and outlook. Eur J Paediatr Dent., 10: 29.

18. Cannon M, Wagner C, Thobaben J, Jurado R and Solt D. (2011): Early response of mechanically exposed dental pulps of swine to antibacterial-hemostatic agents or diode laser irradiation. J Clin Pediatr Dent., 35: 271-276.

19. Basso FG, Pansani TN, Turrioni APS, Bagnato VS, Hebling J and de Souza Costa CA. (2012): In vitro wound healing improvement by low-level laser therapy application in cultured gingival fibroblasts. Int J Dent.

20. Martens L. (2011): Laser physics and a review of laser applications in dentistry for children. Eur Arch Paediatr Dent., 12: 61-67.

21. Golpayegani MV, Ansari G and Tadayon N. (2010): Clinical and radiographic success of low level laser therapy (LLLT) on primary molars pulpotomy. Res J Biol Sci., 5: 51-55.

22. Durmus B and Tanboga I. (2014): In vivo evaluation of the treatment outcome of pulpotomy in primary molars using diode laser, formocresol, and ferric sulphate. Photomed Laser Surg., 32: 289-295.

23. Fernandes AP, Lourenço Neto N, Teixeira Marques NC, Silveira Moretti AB, Sakai VT, Cruvinel Silva T, Andrade Moreira Machado MA, Marchini Oliveira T. (2015): Clinical and radiographic outcomes of the use of Low-Level Laser Therapy in vital pulp of primary teeth. Int J Paediatr Dent. 25: 144-150.

24. Marques NC, Neto NL, Rodini Cde O, Fernandes AP, Sakai VT, Machado MA, Oliveira TM. (2015): Low-level laser therapy as an alternative for pulpotomy in human primary teeth. Lasers Med Sci., 30: 1815-1822.

25. Uloopi K, Vinay C, Ratnaditya A, Gopal AS, Mrudula K and Rao RC. (2016): Clinical evaluation of low level diode laser application for primary teeth pulpotomy. J Clin Diagn Res., 10: ZC67.

26. Odabaş ME, Bodur H, Barış E and Demir C. (2007): Clinical, radiographic, and histopathologic evaluation of $\mathrm{Nd}$ : YAG laser pulpotomy on human primary teeth. J. Endod., 33: 415-421.

27. Frankl S. (1962): Should the parent remain with the child in the dental operatory? J Dent Child., 29: 150-163.
28. Erdem AP, Guven Y, Balli B, Ilhan B, Sepet E, Ulukapi I, Aktoren O. (2011): Success rates of mineral trioxide aggregate, ferric sulfate, and formocresol pulpotomies: a 24-month study. Pediatr Dent., 33: 165-170.

29. Agamy HA, Bakry NS, Mounir MM and Avery DR. (2004): Comparison of mineral trioxide aggregate and formocresol as pulp-capping agents in pulpotomized primary teeth. Pediatr Dent., 26: 302-309.

30. Farsi N, Alamoudi N, Balto K and Mushayt A. (2005): Success of mineral trioxide aggregate in pulpotomized primary molars. J Clin Pediatr Dent., 29: 307-311.

31. Sonmez D, Sari S and Çetinbaş T. (2008): A comparison of four pulpotomy techniques in primary molars: a long-term follow-up. J. Endod., 34: 950-955.

32. Lin P-Y, Chen H-S, Wang Y-H and Tu Y-K. (2014): Primary molar pulpotomy: a systematic review and network meta-analysis. J. Dent., 42: 1060-1077.

33. Vij R, Coll JA, Shelton P and Farooq NS. (2004): Caries control and other variables associated with success of primary molar vital pulp therapy. Pediatr Dent., 26: 214-220.

34. Sun G and Tunér J. (2004): Low-level laser therapy in dentistry. Dent Clin North Am., 48: 1061-1076, viii.

35. Eslamian L, Borzabadi-Farahani A, Hassanzadeh-Azhiri A, Badiee MR and Fekrazad R. (2014): The effect of 810$\mathrm{nm}$ low-level laser therapy on pain caused by orthodontic elastomeric separators. Lasers Med Sci., 29: 559-564.

36. AlGhamdi KM, Kumar A and Moussa NA. (2012): Lowlevel laser therapy: a useful technique for enhancing the proliferation of various cultured cells. Lasers Med Sci., 27: 237-249.

37. Saltzman B, Sigal M, Clokie C, Rukavina J, Titley K and Kulkarni G. (2005): Assessment of a novel alternative to conventional formocresol-zinc oxide eugenol pulpotomy for the treatment of pulpally involved human primary teeth: diode laser-mineral trioxide aggregate pulpotomy. Int. J. Paediatr. Dent., 15: 437-447.

38. Laakso L, Richardson C and Cramond T. (1993): Factors affecting low level laser therapy. Aust J Physiother., 39: 95-99.

39. Gupta G, Rana V, Srivastava N and Chandna P. (2015): Laser Pulpotomy-an effective alternative to conventional techniques: a 12 months Clinicoradiographic study. Int J Clin Pediatr Dent., 8: 18.

40. Niranjani K, Prasad MG, Vasa AAK, Divya G, Thakur MS and Saujanya K. (2015): Clinical Evaluation of Success of Primary Teeth Pulpotomy Using Mineral Trioxide Aggregate ${ }^{\circ}$, Laser and BiodentineTM-an In Vivo Study. J Clin Diagn Res., 9: ZC35.

41. Ansari G, Morovati SP and Asgary S. (2018): Evaluation of four pulpotomy techniques in primary molars: A randomized controlled trial. Iran. Endod. J., 13: 7. 